ALPHA Nº 28 Julio 2009 (105-117)

ISSN 0716-4254

http://alpha.ulagos.cl

\title{
SOBRE LA COHERENCIA Y LAS IMPLICANCIAS DE UN LIBERALISMO COHERENTE NO MULTICULTURALISTA
}

On coherence and the implications of a non-multiculturalist coherent liberalism

Carlos Acevedo Rodríguez*

\section{Resumen}

En este ensayo se argumenta la incoherencia lógica de asociar los principios del pensamiento liberal con el desarrollo de propuestas de raigambre multiculturalista, y se señalan las implicaciones últimas que conlleva el ser un liberal coherente. Para tal acometido, primero, se indican las contradicciones de dos autores multiculturallistas que intentan, a la vez, denominarse liberales (Kymlicka y Walzer) y, segundo, se analizan los supuestos de un autor liberal que rechaza los planteamientos multiculturalistas (Barry).

Palabras clave: Liberalismo, multiculturalismo, ciudadanía diferenciada, igualdad compleja, liberalismo igualitario, represión liberal.

\section{Abstract}

This essay discusses the logical in incoherence associating liberal thought with the development (of multiculturalist proposals, and it identifies the latest implications of being a coherent liberal. For this undertaking, first, we show the contradictions of two multiculturalists authors who also try to call themselves liberal, (Kymlicka and Walzer), and second, we analyze the consequences of one liberal author that rejects multiculturalists approaches (Barry).

Key words: Liberalism, multiculturalism, differentiated citizenship, equality complex, liberalism egalitarian, liberal repression.

\section{INTRODUCCIÓN}

Cuando pensamos en los principios liberales de justicia, igualdad y libertad, debemos siempre tener en cuenta que éstos tienen su origen en los derechos del hombre promovidos por la revolución francesa y fortalecidos por la revolución norteamericana. Estos principios han dado forma a las democracias constitucionales, a la ciudadanía, y han configurado el propio paradigma de lo que hoy conocemos como derechos universales.

Si bien estos principios tienen un origen histórico concreto, los liberales los dotan de un contenido universal de modo que, evidentemente, estos 


\section{Carlos Acevedo Rodríguez}

principios asumen una supremacía moral en cuanto devienen en la expresión última de la potencialidad y del respeto de la persona humana y, por ende, se constituyen en máxima que pueden dirigir a la humanidad hacia la paz perpetua. Ahora bien, el problema de un liberalismo incoherente lo enfrentamos cuando autores que se autodenominan liberales intentan conciliar los derechos universales con los derechos de culturas y comunidades étnicas concretas, o lo que se denominan derechos de grupo.

Un liberal asume los principios culturales como universales (no como un avance particular de un tipo específico de cultura como es nuestra idea). No valora, ni tampoco aprehende ni comprende, los elementos diferenciadores de una cultura concreta, en tanto no sea para señalar los grados en que se alcanzan los principios liberales en ésta. En consecuencia, una cultura es valorada en forma positiva, única y exclusivamente si está acorde con los principios liberales. De esta manera, el multiculturalista de raigambre liberal puede transitar por caminos contradictorios cuando a la luz de los principios liberales intenta rescatar el particularismo, muchas veces antiliberal, de comunidades étnicas. Es pues, un vaivén entre valorar lo universal y lo concreto en su especificidad, lo cual más que desorientador resulta contradictorio.

En lo que sigue hacemos hincapié en las contradicciones en que caen dos autores que podríamos denominar liberales multiculturales para, luego, enfrentarlos con el pensamiento de un liberal coherente, concluyendo, finalmente, con una reflexión de las últimas consecuencias lógicas de la posición liberal.

\section{EL ARGUMENTO DE WILL KYMLICKA}

Kymlicka, en Ciudadanía multicultural (1996), diferencia entre Estados multinacionales y Estados poliétnicos. Los primeros corresponden a Estados donde confluyen un conjunto de culturas o naciones que comparten una historia, lenguaje y tierra originaria en común y que buscan la sobrevivencia como comunidad cultural. Los segundos hacen referencia a distintos componentes étnicos que, por medio de la migración, han conformado un territorio donde estos componentes buscan la integración a la comunidad cultural total. Por tanto, cuando Kymlicka habla de un Estado multicultural se refiere a un Estado que es multinacional, poliétnico, o ambos.

Como buen multiculturalista, Kymlicka propone una serie de derechos específicos que vienen dados por pertenecer a una comunidad cultural étnica. Según él, al menos existen tres formas de derechos específicos en función de la pertenencia grupal: 1) Derechos de autogobierno, 2) Derechos poliétnicos, 3) Derechos especiales de representación (1996:47). 
Los primeros derechos hacen referencia a poderes políticos de autogobierno, los segundos a exenciones de ciertas leyes y los terceros se refieren a representación de los grupos más desfavorecidos.

Cuando el derecho de representación es un corolario del autogobierno, se debe asegurar la representación en cualquier organismo que pueda interferir en las mismas competencias de autogobierno. Si se busca la autonomía de las culturas en relación al autogobierno (como a primera vista parecería proponer Kymlicka) sus representaciones deben ser permanentes. Si preguntamos por cuáles son los grupos que tienen derecho al autogobierno se nos responde que sólo las minorías nacionales tienen los derechos de reivindicar el autogobierno.

En el desarrollo de tales derechos grupales, Kymlicka trae a colación el concepto de ciudadanía diferenciada que — por lo demás, para él— nada tiene de contradictorio con los principios liberales de igualdad y libertad. Para probar esta afirmación, Kymlicka realiza la distinción entre restricciones internas y protecciones externas de un grupo. Las restricciones internas se refieren a las relaciones intragrupales que ocurren cuando el grupo coacciona a sus miembros para que mantengan el consenso hacia creencias y tradiciones arraigadas. Las protecciones externas se basan en las relaciones intergrupales orientadas a proteger al grupo de interferencias externas que atenten contra su propia dinámica interna como grupo.

Cuando hablamos de las restricciones internas de un grupo debe quedar claro que, dada la vertiente liberal de Kymlicka, se debe proteger a los individuos del grupo en los que se intente imponer derechos no liberales o de instituciones no democráticas que busquen imponerse en nombre de la tradición cultural o de la ortodoxia religiosa. En este sentido, se puede decir que las restricciones aluden a los casos en "donde las libertades civiles y políticas básicas de los miembros del grupo se ven restringidas” (1996:53). A la vez, las protecciones externas se dirigen a mantener la "identidad" del grupo y su supervivencia con respecto a las interferencias externas de la sociedad de la que forma parte. Entonces, el argumento es que tales protecciones externas deben ir a favor de grupos que no buscan imponer restricciones internas sobre sus miembros, es decir, a éstos no se les impide dirigir la mirada a "cuestionar y a revisar las autoridades y las prácticas tradicionales” (1996:60).

Así, los tres derechos diferenciales de grupo (poliétnicos, representación y autogobierno) se basan en las protecciones externas de grupos "razonables" que lo único que buscan es "asegurarse de que el conjunto de la sociedad no les privará de las condiciones necesarias para su supervivencia, ni controlar la medida en que sus propios miembros adhieren a prácticas poco tradicionales u ortodoxas”, según Kymlicka (1996:61). Frente a tal 


\section{Carlos Acevedo Rodríguez}

argumentación ¿ Se puede (y en qué grado) hacer lo uno sin lo otro? Es decir, en qué medida la pervivencia de un grupo étnico depende única y exclusivamente de la protección en contra de los no miembros del grupo, en tanto ninguno de éstos puede realizar decisión que afecte a la dinámica interna del grupo, cuando es evidente que todo grupo condiciona a sus miembros para que se dirijan al consenso, es decir, todo grupo impone restricciones internas. Ya sea la familia, el lenguaje o la posición en la estructura social, hay una gran variedad de elementos que son necesarios en un proceso de socialización (primario y secundario) que moldean los habitus de los individuos configurando sus propios esquemas de percepción y de acción. ¿En qué tipo de comunidad piensa Kymlicka? Pues, piensa en una comunidad que a todas luces busca la integración hacia una cultura liberal. Kymlicka — como buen liberal y como multiculturalista a medio camino - sigue manteniendo la supremacía de la sociedad moderna y argumenta que es necesario "limitar la libertad de los que no pertenecen al grupo, restringiendo su capacidad de tomar decisiones económicas o políticas relativas a la comunidad minoritaria y a los recursos de la misma” (1996:62) mientras, claro está, la idea que se desprende es que evidentemente tal límite no debe sobrepasar los derechos liberales, pues, en el caso que el grupo niegue o rompa tales derechos sí debe haber intervención de no miembros. De este modo, estamos frente a una "teoría” que sólo se refiere a grupos que buscan la integración a la sociedad liberal, grupos que mantienen cosmovisiones "razonables" y que sólo en referencia al seguimiento de los principios liberales se puede otorgar o no otorgar valía a las culturas de estos grupos. Y, así, es claro que las comunidades que no son valorables en tanto no se acoplan a los principios liberales no caben dentro de tal teoría de la ciudadanía diferenciada y de los derechos de minoría; están al otro lado de la humanidad liberal; son los extraños que no entran en el mundo teórico de la tolerancia. De esta forma, la cuestión se basa en por qué Kymlicka defiende derechos multiculturales si lo que se busca es una integración liberal. Un liberal no totalmente coherente que se oculta tras un velo multicultural para no argumentar las cosas como realmente son: integración liberal y sólo ésta.

En este sentido, Kymlicka argumenta que otorgar derechos grupales a una comunidad implica conocer a fondo la medida en que tales protecciones pueden atentar contra las libertades liberales de los miembros del grupo. Empero, y debido a que la fuente multiculturalista sigue latiendo, si aceptamos derechos grupales que Kymlicka propone, tales como protecciones lingüísticas, territoriales y educacionales (como en Quebec), es evidente que se establecen restricciones internas a los individuos para poder decidir abandonar su comunidad (cualquier ciudadanía diferenciada establece restricciones). En consecuencia, si aceptamos que "el objetivo de las proteccio- 
nes externas es asegurar que la gente pueda mantener su forma de vida si así lo desea, así como que las decisiones ajenas a la comunidad no les impidan hacerlo”, según sostiene Kymlicka (1969:67, cita 11) es totalmente legítimo preguntarse — como ya lo hemos hecho- por las condiciones reales de elección de un individuo que nace en un grupo con derechos diferenciales de su comunidad (territorial, educacional o lingüística). He aquí a un liberal que no puede ser coherente en tanto sigue palpitando su anhelo multiculturalista. Anhelo, que por lo demás, parece haber olvidado las enseñanzas de la antropología estructural, la que esclarece el peligro que plantea la modificación de un elemento cultural cualquiera en una configuración de la cultura como sistema interconectado e interdependiente.

\section{EL ARGUMENTO DE MICHAEL WALZER}

En Las esferas de la justicia (1993) Walzer propone una muy interesante visión técnica de la justicia basada en la igualdad compleja. Para Walzer, la justicia se asienta en un contexto histórico particular y se refiere al sentido propio de los bienes en tanto develan lo justo o injusto de su distribución. Si tales significados son distintos, las distribuciones deben basarse en un criterio autónomo. Es decir, como cada criterio de distribución de un bien corresponde a la esfera particular en que cobra sentido el propio bien, no debe interferir la lógica de la distribución de tal bien con la lógica de distribución de otra esfera en que tiene sentido otro tipo distinto de bien. Así, por ejemplo, el dinero que funciona en la esfera del mercado no debe interferir en las restantes esferas que tienen bienes particulares. Dicho de otra manera, tener dinero no debe ser un recurso para tener amor, prestigio, representación política, etc. Ningún bien debe ser dominante y, por ende, ningún bien debe ser tal que pueda adquirir poder e intercambiarse por otros bienes en otras esferas que escapan a su propia lógica.

Cuando se cuestiona tal convertibilidad y se plantea la autonomía relativa de las distintas esferas se cuestiona el propio predominio. En este sentido, cada bien en su esfera particular concreta puede generar desigualdades y monopolios que no serían injustos en tanto no sean convertibles en la adquisición de poder en otras esferas. Y es aquí, precisamente, cuando se da la igualdad compleja.

En contraste, cuando lo que se cuestiona es el monopolio y no el predominio, se propone una igualdad simple. La igualdad simple se basa en redistribuir equitativamente algunos bienes dominantes. Empero, tal igualdad produce nuevas desigualdades, pues, al no ser ya dominante ese bien redistribuido, se producen nuevas desigualdades con respectos a otros bienes que adquieren nuevo valor y devienen en dominantes. En caso de que el 


\section{Carlos Acevedo Rodríguez}

Estado interfiera para que no se produzcan tales nuevas desigualdades y monopolios, es el mismo poder estatal el que estará ahora en juego. Esto nace, entonces, de la perspectiva de cuestionar el monopolio, mas no el predominio, siendo claro que para alcanzar la igualdad compleja, cada bien debe manejarse dentro de su esfera particular.

Ahora bien, nuestra crítica a esta teoría argumenta que ella se refiere únicamente a sistemas altamente diferenciados en sus funciones que caracterizan a la sociedad moderna (y tan sólo a ésta). Por lo mismo, si se asume la existencia de sociedades donde el predominio y el monopolio no son violaciones sino observancia de los significados, tales como en la Edad media y en la sociedad de castas hindú, Walzer se ve en la obligación de argumentar que "mientras más perfecta sea la cohesión menos se podrá pensar en la igualdad compleja” (1993:40). Todo se complica aún más cuando en Politics and passions (2004) Walzer muestra el espíritu multicultural y argumenta en favor de los derechos culturales. Se complica, decimos, en tanto en este texto se apela por la intervención del Estado en cuanto a mantener derechos grupales que se caracterizan por una pertenencia involuntaria. Si bien Walzer (en una visión mucho más clara que Kymlicka) establece la importancia del grupo cultural como un elemento que condiciona tanto las acciones y decisiones de los sujetos —así como la asociación de éstos a grupos voluntarios - argumenta que el Estado debe intervenir para sostener la reproducción de tales grupos culturales en la mantención de sus propios lenguajes y currículas. Como ya es evidente, en este rol protector de la reproducción cultural de los grupos el Estado debe resguardar que se otorgue una educación siempre democrática y debe proteger a grupos que no presenten un peligro para la misma democracia. Así, se establece que los grupos que atentan contra la democracia no deben ser protegidos y, por ende, deben ser rechazados. Ante tal acomodo de culturas a los principios liberales, nos podemos preguntar por la división de las esferas, y cuestionarlos por el argumento de que la justicia que deviene de los monopolios y desigualdades se mantiene siempre y cuando los bienes de cada esfera no sean convertibles a los de otra. En consecuencia, es claro que la justicia de la autonomía relativa de las esferas y la no intervención de una esfera hacia la otra es únicamente válida para grupos democráticos, pues, en tanto hablamos de grupos no democráticos, la esfera de la política liberal sí debe intervenir en las esferas no liberales y "no justas" de las comunidades étnicas y transformar, así, el mismo sentido de los bienes sociales que ha construido una comunidad. Con este proceder, sin embargo, se torna nuevamente al Estado como un objetivo de lucha por su monopolio y se retorna a una igualdad simple. Y, entonces, así es como vemos de nuevo la inconsistencia de un multiculturalismo (si 
entendemos por éste el respeto de distintas cosmologías) cuando quiere ser, a la vez, liberal.

\section{EL ARGUMENTO LIBERAL COHERENTE Y SIN MÁSCARA DE BRIAN BARRY}

En Culture ecuality (2001) Brian señala que los derechos que vienen dados en función de un grupo son derechos individuales, no derechos grupales. Si se puede hablar en un sentido adecuado de derechos grupales, se debe hacer en cuanto los derechos afectan a todos los miembros del grupo, pero, siempre y sin excepciones, los miembros del grupo son libres tanto de seguir como de no aceptar tales derechos; en ningún caso, pues, el grupo tiene primacía sobre el individuo.

Ahora bien, cuando se está frente a grupos que sufren privaciones, se debe atacar la privación en cuanto tal y al conjunto de los grupos que sufren tales carencias. En esta perspectiva, lo que se ataca es la privación en tanto privación y no como un derecho diferencial de grupo alguno. Entonces, sobre la base del "liberalismo igualitario" se debe subsanar cualquier desigualdad de la cual el individuo no es responsable. En este sentido, en el liberalismo igualitario se apela a medidas especiales de asistencia en favor de los miembros de grupos en desventaja o de bajos ingresos, pobre calidad de las viviendas, carencia de un trabajo (o un trabajo que no paga lo suficiente para vivir); pobreza de educación, alta probabilidad de ser víctimas de violencia física, un ambiente malsano y así sucesivamente. Barry sostiene que las políticas dirigidas a quienes sufren de estas y otras formas de privación son incuestionadamente justificables y de hecho moralmente necesarias (2001: 114).

En consecuencia, Barry no se enfoca sobre el problema del grupo cultural étnico en sí mismo, sino que establece una visión abarcadora que integra a todo grupo marcado por alguna privación. Cuando se actúa con base en el grupo y no con base en la privación, se actúa con políticas bajo inclusivas, marginando a grupos que sufren de igual privación. En cambio, cuando se sigue la intervención por la privación, se actúa con políticas sobre inclusivas. Estas últimas políticas (y sólo éstas) se pueden denominar equitativas.

Lo que se busca con las políticas sobre inclusivas, corresponde directamente a la inclusión, por ende, tales políticas se deben ir eliminando a medida que se consiga tal objetivo. De modo alguno deben ser políticas permanentes, ni se deben aceptar argumentos multiculturalistas que apelen a la mantención de la diferencia cultural en forma permanente. Los multiculturalistas no apelan por la igualdad de oportunidades, sino que - $-\mathrm{y}$ en el 


\section{Carlos Acevedo Rodríguez}

momento de conseguir tal igualdad - siguen rebatiendo con argumentos culturales de demandas comunitarias especiales. Según Barry, "mientras el liberalismo igualitario no excluye el tratamiento especial para los miembros de ciertos grupos, lo que éste realmente lamenta es precisamente el tratamiento especial pedido por el multiculturalista” (2001:117).

La crítica que hacen los multiculturalistas al liberalismo se basa en que los grupos que siguen los principios liberales están en mejores condiciones de conseguir sus fines en contraste con aquellos que no los siguen. Pero Barry argumenta que la promesa del liberalismo es la de otorgar un tratamiento igual a los individuos; no a grupos liberales y no liberales. Así, el énfasis del liberalismo sobre el bienestar individual no es negociable, puesto que es éste el principio en el cual se debe limitar la libertad de los grupos.

Ahora bien, los principios liberales de libertad e igualdad que — según Barry- encuadran las libertades constitucionales de "igualdad civil, libertad de habla y de religión, no discriminación, oportunidades equitativas, y así sucesivamente” (2001:122), en todo momento son el principio regulador de un liberalismo igualitario que acepta, a la vez, la diversidad y a la autonomía. Este tipo de liberalismo deja un gran espacio a la decisión individual y a la diversidad. Se pueden elegir creencias, prácticas, grupos, etc. Siempre y cuando éstos se hallen en el marco del respeto de los principios liberales y no atenten contra los derechos universales de los seres humanos. En esta visión, la autonomía es de suma importancia en cuanto permite, mediante la promoción de parte del Estado, la reflexión y la puesta en cuestión de las propias creencias y prácticas. La confluencia de diversidad y autonomía permite la pertenencia voluntaria a los grupos de los que se forma parte, mientras que, en el marco de los principios liberales, ningún grupo puede negar la salida de sus miembros y la sanción máxima que una comunidad puede establecer sobre algún miembro es la expulsión, y tan sólo la expulsión. Si bien es verdad que tenemos grupos involuntarios y sanguíneos, el Estado se debe asegurar que se guíen bajo los principios liberales y debe crear, a la vez, instituciones que puedan recibir al individuo afectado por cualquier tipo de coacción y que sean capaces de proteger al individuo de cualquier tipo de marginación en su integración a la sociedad.

Conforme a lo dicho, la dinámica de cualquier grupo debe estar limitada por los principios liberales de igualdad y libertad. Por tanto, no se les puede otorgar medidas especiales a ningún grupo o líder para que viole los derechos individuales, ni se puede fortalecer a los grupos en contra de sus miembros. Si bien el liberalismo es tolerante, se trata de una visión de la tolerancia con toda norma que no contravenga los principios liberales.

En Barry se aceptan sin problema alguno las consecuencias lógicas de la perspectiva liberal que se asienta sobre esta visión de la tolerancia. Estas 
consecuencias se expresan en que cuando los grupos "contravienen los cánones de equidad y respeto pueden legítimamente ser reprimidos por un Estado liberal” (2001:127). Todas las sanciones, repetimos, que un grupo pueda realizar en sus miembros deben estar limitadas por los principios culturales de libertad e igualdad. En este sentido, Barry argumenta que el liberalismo no puede acomodarse a la diversidad profunda (2001:128).

Hablar de una especie de relativismo moral es hablar de principios que atentan contra los derechos liberales básicos. El relativismo moral no puede ser aceptado frente a los derechos liberales universales. Por lo tanto, los principios liberales, en ningún momento, deben ser cuestionados en su universalidad, pues, hacerlo (como lo hace el relativismo moral) sería rebajarlos a la categoría de prejuicios particulares. El problema de la diversidad es, entonces, un problema de grados. Como afirma Barry, "la diversidad es deseable en grados, y sólo en grados, en que cada uno de los diversos grupos funciona de una manera en la que está bien adaptado al avance y la seguridad de los derechos de sus miembros. Pero esta es precisamente la posición liberal” (2001:134). Una cultura, entonces, se valora en referencia a su capacidad para respetar los derechos humanos, es decir, se valoran en referencia a un marco de principios universales. De esta manera, se puede decir que aquellos modos de vida que son compatibles con las instituciones liberales no están amenazados por tales instituciones. Por lo tanto, los únicos modos de vida que necesitan apelar al valor de la diversidad cultural son aquellos que necesariamente involucran inequidades injustas o requieren poderes de adoctrinamiento y de control en orden a mantenerse a sí mismas incompatibles con el liberalismo. Desde el momento que semejante cultura sea injusta y opresiva con el último de sus miembros, es difícil ver por qué ella debería mantenerse viva artificialmente (2001:135).

Ahora podemos pasar a las consecuencias lógicas del liberalismo coherente. En tanto el buen liberal es universal no puede permitir que se violen los principios liberales en ninguna parte del planeta. Todo el mundo, pues, debe encontrarse bajo la protección de las instituciones liberales. Y el buen liberal debe asegurar la paz a la que le dirige los principios liberales, justamente, promoviendo la intervención para prevenir la opresión y la injusticia. En consecuencia, estamos frente a la idea de que una guerra dirigida a consolidar la paz y los derechos humanos (algo tan común actualmente) es una guerra justa (en tanto evita un mal mayor). Si bien en este punto es claro, tanto la lógica liberal así como el fin que se desprende de ésta (encaminarse hacia la paz perpetua en que se respeten los derechos universales en todo el planeta), Barry sostiene que "puede ser absolutamente incierto a veces cuáles son los mejores medios a tal efecto. Por lo menos, los liberales no son tan simples para imaginarse que la respuesta a todas las 


\section{Carlos Acevedo Rodríguez}

violaciones del derecho liberal será enviando a los marines, o aún una fuerza de Naciones Unidas. Como en cualquier teoría de la guerra justa, debe haber una doctrina de la "proporcionalidad" (2001:138). Una doctrina de la proporcionalidad, entonces, que establezca la justa medida de violencia a utilizar para oprimir el "mal" (doctrinas y acciones no liberales que violan los derechos humanos) en cuestión.

\section{CONCLUSIÓN}

En este breve desarrollo sobre las consecuencias lógicas del argumento liberal coherente, hemos querido hacer notar la intolerancia que está presente en el liberalismo en cuanto se afirma en principios universalistas que son vistos como los derechos universales que se han desprendido de todo particularismo histórico. Frente a tal idea liberal, se ha llamado la atención sobre la incongruencia del intento de conciliar tales principios universales con el rescate de comunidades culturales, pues, en tanto las comunidades no respeten tales principios (ajenos a su historia) deben ser rechazadas, no valoradas, intervenidas y hasta combatidas. De este argumento no se puede escapar ningún liberal, por muy multiculturalista que se piense.

De este modo, al mismo tiempo que desarrollamos el argumento de que frente a la intolerancia liberal hacia comunidades que quebrantan los derechos liberales se torna incoherente conciliar la perspectiva multicultural con el liberalismo, también se expuso el tipo de argumentación de un liberal coherente que, por ser coherente, rechaza explícitamente los argumentos multiculturales. Con tal argumentación no supusimos ningún valor superior de los principios liberales y no se asumió la postura de ninguno de los autores expuestos.

Ahora bien, primero, haciendo justicia al liberalismo, debemos mencionar que podemos pensar en éste como un modelo normativo a seguir; como un ejercicio heurístico que sirva como marco de referencia al modelo óptimo de sociedad en que los sujetos se reconocerían unos con los otros bajo una actitud reflexionada, enmarcada en los principios que exaltan la potencialidad del ser humano y encaminada al consenso. Segundo, desbordando el multiculturalismo liberal y rescatando las visiones del relativismo cultural, debemos señalar que los derechos liberales corresponden únicamente a una vía cultural de las restantes existentes y por existir y, por ende, no debemos tomar tal marco como valoración de culturas y comunidades extrañas a esta historia particular.

En tal debate no se pueden perder de vista dos elementos: a pesar de los intentos que pretenden establecer la universalidad de los principios liberales, ya sea sobre la base de un contractualismo imaginario en el cual los sujetos 
razonables, mediante un velo de la ignorancia, estableciesen en conjunto y por consenso tales principios (Rawls, 1995) o argumentando la universalidad de los principios basándose en acciones comunicativas guiadas a poner en cuestión y poder defender los argumentos de validez en referencia tanto a una comunidad trascendental como a una comunidad concreta (Habermas, 1998) todo constructo y norma social establecida presenta un carácter histórico; y el modelo normativo que estos principios ostentan están muy lejos de realizarse en nuestras sociedades que siguen estando basadas en relaciones de dominación, exclusión y asimetrías en el acceso a los derechos liberales.

Tanto el liberal coherente (aquel que se interesa por atacar la privación de grupos e individuos en tanto privación, no llamando a derechos culturales especiales, sino que pensando en la realización del marco normativo de los principios universales en la sociedad total), así como el liberal multiculturalista; pueden asumir tan sólo la segunda parte de nuestro enunciado. Lo peligroso del universalismo del marco normativo liberal es que nos lleva al siguiente planteamiento: mientras "nosotros" defendemos los derechos humanos, los "otros" que no los defienden, se hallan en contra de la humanidad misma. En este sentido, incluso el liberalismo multiculturalista (que, dentro de los liberales, se supone el más tolerante de cosmovisiones diversas) ha sido muy claro en manifestar el afán de que se respeten los derechos humanos en todo el mundo, lo cual lleva fácilmente a justificar intervenciones en diversas comunidades, y en un contexto global, a la doctrina de una guerra "justa" así como a la noción de la guerra "preventiva" en aras de la democratización. En tales pensamientos, se arguye estar en el lado y a favor de la "humanidad", lo cual puede degenerar en una guerra injusta y en meras invasiones que "deshumanizan” al "enemigo”. He aquí la problemática que todo liberal debe manejar, principalmente en un mundo en el que existe una gran parte de la población que no se integra a los principios liberales y en las que sus propias normas culturales son fuertemente anti-liberales.

Los autores multiculturalistas revisados, nos indican la incongruencia de no tomar una decisión entre el respeto de los principios universales en todos los rincones del planeta, y entre el respeto de la diversidad y la tolerancia hacia toda comunidad, bajo un esquema de relativismo cultural. Para superar esta elección de tipo suma cero, se deben construir nociones de síntesis más potentes (si esto es posible) que no sean atacables bajo la noción de incoherencia. ${ }^{1}$

\footnotetext{
${ }^{1}$ En contra de una reflexión que pueda construir una síntesis, creemos que las elección entre alternativas polares se torna de mayor fortaleza en procesos históricos como son las situaciones de colonización y de los conflictos bélicos actuales que, si bien tienen una base material y
} 


\section{Carlos Acevedo Rodríguez}

Un paso esencial para tales síntesis, es atacar el trascendentalismo universal y apelar a la historización reflexiva y a la crítica (crítica en sentido kantiano) de nuestras creencias y prácticas. De esta forma, se da cabida a que las comunidades no occidentales, si así lo buscan, puedan elegir principios no liberales que guíen sus acciones. Esto, claro está, se debe permitir mientras no represente un peligro a la violación de los derechos de las restantes comunidades, sean o no liberales. En este marco de pensamiento y en su ejercicio de historización y tolerancia, el liberal debe dirigir todos sus esfuerzos a atacar las privaciones y desigualdades (de las cuales el individuo no es responsable), en las comunidades occidentales: éste, y sólo éste, debe ser su campo de acción. $^{2}$

Si bien no podemos dejar de contentarnos en pensar que al verse cumplido el ideal liberal (en la sociedad o civilización que sea) las relaciones de dominación, exclusión y abuso se verán desplazadas, se debe aceptar que "nuestro punto de vista más querido puede convertirse en una más de las múltiples formas de organizar la vida, importante para quienes están educados en la tradición correspondiente, pero completamente desprovisto de interés y acaso un obstáculo para los demás” (2008:92). Muchas de las contradicciones en las que caemos se pueden superar cuando se esclarece que "sólo unos pocos están satisfechos de poder pensar y vivir de una forma que les agrada, sin soñar en imponer obligatoriamente a los demás su tradición” (2008:92).

mercantil muy clara, el argumento ideológico que se dirige a la población se enmarca en la defensa e instauración de los derechos liberales.

Por otro lado, y pensando en la dificultad que tiene el aceptar el relativismo cultural, es útil establecer la necesidad que tienen los marcos trascendentales de realidades últimas (religiones o ideologías), en las cuales se cumplen los imperativos morales, para poder decir que actuar moral y racionalmente no es contradictorio. $\mathrm{O}$ en otras palabras, que actuar sobre la base de imperativos que en la finitud e imperfección humana nunca serán alcanzados en la realidad cotidiana, no es irracional en tanto serán logrados en una realidad del más allá, Cfr. McCarthy, (1992). En este sentido, es fácil apreciar la resistencia a la posibilidad de asumir como prejuicios morales tales realidades trascendentales y, por ende, pensar en una convivencia pacífica entre éstas. Ambas reflexiones serán objeto de desarrollo en futuros ensayos.

${ }^{2}$ Esta propuesta que adquiere la apariencia de una convivencia en aislamiento de comunidades culturales o, en el mejor de los casos, con interacciones mínimas entre éstas, es un primer paso y como tal es del todo insuficiente. Se debe profundizar en las características del organismo o agencia que asegure la convivencia pacífica y las fronteras; en la problemática que surge cuando algunas comunidades occidentales intenten defender actos ilegales o de violación a los derechos humanos desde la perspectiva de la tolerancia; en la medida, si es que es posible, en la que se debe dar cabida a que individuos y grupos occidentales puedan cambiar de comunidades culturales de forma voluntaria y, muy relevante, argumentar la coherencia lógica que tiene ser liberal en este marco de reflexión. Como se aprecia, quedan muchos vacíos y una enormidad de tareas pendientes. 
Sobre la coherencia y las implicancias de un liberalismo coherente

El Colegio de México, Centro de Estudios Sociológicos (CES)* Camino al Ajusco \# 20. Pedregal de Santa Teresa, Delegación Tlalpan

C.P. 10740 (México D.F.)

desborde@gmail.com

\section{BIBLIOGRAFÍA}

BARRY, Brian. Culture \& ecuality. Massachusetts: Harvard University Press Cambridge, 2001.

FEYERABEND, Paul. La ciencia en una sociedad libre. Distrito Federal: Siglo Veintiuno, 2008.

HABERMAS, Jürgen. Facticidad y validez. Madrid: Trotta, 1998.

KYMLICKA, Will. Ciudadanía multicultural. Una teoría liberal de los derechos de las minorías. Barcelona: Paidós, 1996.

MCCARTHY, Thomas. Ideales e ilusiones. Reconstrucción y deconstrucción en la teoría crítica contemporánea. Madrid: Tecnos, 1992.

RAWLS, John. Teoría de la justicia. Ciudad de México: F. C. E., 1995.

WALZER, Michael. Las esferas de la justicia. Una defensa del pluralismo y la igualdad. Ciudad de México: F. C. E., 1993.

Politics and passions: Toward a more egalitarian liberalism. New Haven: Yale University Press, 2004. 